\title{
Integrin engagement regulates monocyte differentiation through the forkhead transcription factor Foxp1
}

\author{
Can Shi, ${ }^{1}$ Xiaobin Zhang, ${ }^{1}$ Zhiping Chen, ${ }^{1}$ Karina Sulaiman, ${ }^{1}$ Mark W. Feinberg, ${ }^{1}$ \\ Christie M. Ballantyne, ${ }^{2}$ Mukesh K. Jain, ${ }^{1}$ and Daniel I. Simon ${ }^{1}$ \\ ${ }^{1}$ Cardiovascular Division, Brigham and Women's Hospital, Harvard Medical School, Boston, Massachusetts, USA. ${ }^{2}$ Department of Medicine, \\ Baylor College of Medicine, Houston, Texas, USA.
}

\begin{abstract}
The precise signals responsible for differentiation of blood-borne monocytes into tissue macrophages are incompletely defined. "Outside-in" signaling by integrins has been implicated in modulation of gene expression that affects cellular differentiation. Herein, using differential display PCR, we have cloned an 85-kDa forkhead transcription factor (termed Mac-1-regulated forkhead [MFH] and found subsequently to be identical to Foxp1) that is downregulated in $\beta_{2}$-integrin Mac-1-clustered compared with Mac-1-nonclustered monocytic THP-1 cells. MFH/Foxp1 is expressed in untreated HL60 cells, and its expression was markedly reduced during phorbol ester-induced monocyte differentiation, but not retinoic acid-induced granulocyte differentiation. Overexpression of $M F H / F o x p 1$ markedly attenuated phorbol ester-induced expression of $c$-fms, which encodes the M-CSF receptor and is obligatory for macrophage differentiation. This was accompanied by decreased CD11b expression, cell adhesiveness, and phagocytosis. Using electromobility shift and reporter assays, we have established that MFH/Foxp1 binds to previously uncharacterized sites within the $c$-fms promoter and functions as a transcriptional repressor. Deficiency of Mac-1 is associated with altered regulation of $M F H$ / Foxp1 and monocyte maturation in vivo. Taken together, these observations suggest that Mac-1 engagement orchestrates monocyte-differentiation signals by regulating the expression of the forkhead transcription repressor $M F H / F o x p 1$. This represents a new pathway for integrin-dependent modulation of gene expression and control of cellular differentiation.
\end{abstract}

\section{Introduction}

Pluripotent hematopoietic stem cells undergo progressive restriction in their lineage potential to give rise to mature terminally differentiated cells. The process of hematopoietic differentiation is thought to follow a developmentally ordered pattern of gene expression. Transcription factors play a key role in the lineage determination and maturation of hematopoietic cells (1). Identification of these regulators and determination of the mechanism of how they activate their target genes are important for understanding the development of monocytes and macrophages. For example, the $c$-fms gene that encodes for the M-CSF receptor is regulated by the coordinated effects of three transcription factors, PU.1, AML1 $(\mathrm{CBF} \alpha 2)$, and CCAAT enhancer-binding protein (C/EBP) (2). This critical receptor is required for the differentiation, proliferation, and survival of monocytic phagocytes. Deficiency of c-fms is associated with large depletions of macrophages in most tissues $(3,4)$.

However, the precise external signals that control differentiation of peripheral blood monocyte to tissue macrophage are incompletely defined. Monocytes leave the bone marrow and travel through peripheral blood vessels. Once they reach a tissue, perhaps in response to M-CSF, GM-CSF, monocyte chemoattractant pro-

Nonstandard abbreviations used: activator protein-1 (AP-1); all-trans retinoic acid (RA); binding site (bs); CCAAT enhancer-binding protein (C/EBP); differential display PCR (DD-PCR); electrophoretic mobility shift assay (EMSA); Mac-1-regulated forkhead (MFH); murine stem cell virus (MSCV); phycoerythrin (PE); rapid amplification of cDNA ends (RACE); terminal deoxynucleotidyl transferase (TdT).

Conflict of interest: The authors have declared that no conflict of interest exists.

Citation for this article: J. Clin. Invest. 114:408-418 (2004)

doi:10.1172/JCI200421100. tein-1 (MCP-1), and/or IL-3, they differentiate into macrophages by growing in size and increasing their lysosomal compartment, the amount of hydrolytic enzymes, the number and size of mitochondria, and the extent of their energy metabolism (5).

Receptors of the integrin family mediate adhesion of cells to extracellular matrices as well as intercellular interactions that are central to inflammation, immunity, hemostasis, and tumor metastasis (6). These adhesive interactions transduce "outside-in" signals that control complex cell functions, such as proliferation, differentiation, and survival, and require the regulation of gene expression (7). Neutrophil and monocyte recruitment in acute inflammation is mediated in part by the $\beta_{2}$-integrin family of receptors, LFA- 1 $\left(\alpha_{\mathrm{L}} \beta_{2}, \mathrm{CD} 11 \mathrm{a} / \mathrm{CD} 18\right), \mathrm{Mac}-1$ ( $\left.\alpha_{\mathrm{M}} \beta_{2}, \mathrm{CD} 11 \mathrm{~b} / \mathrm{CD} 18\right)$, and $\mathrm{p} 150,95$ $\left(\alpha_{X} \beta_{2}\right.$, CD11c/CD18). Engagement of $\beta_{2}$-integrins by a broad repertoire of ligands generates "outside-in" signals, leading to inflammatory cell activation and induction of genes encoding for IL-1 $\beta$, TNF- $\alpha$, and tissue factor $(8,9)$. While $\beta_{2}$-integrins have been implicated in modulation of activator protein-1 (AP-1) and NF- $\kappa$ B activity (10), the signal transduction pathways have been elucidated only recently $(11,12)$.

We have shown that mAb blockade (13) or absence (14) of Mac-1 modulates the biological response to vascular injury, reducing vessel wall inflammation and neointimal thickening after experimental angioplasty. This led us to hypothesize that clustering and activation of Mac-1 may initiate a novel gene program that promotes vascular inflammation. Our goal was to identify genes regulated by Mac-1 and to characterize these signaling pathways. Gene transcription was analyzed using differential display PCR (DDPCR) from Mac-1-clustered compared with Mac-1-nonclustered 
monocytic THP-1 cells. Herein, we have cloned an $85-\mathrm{kDa}$ forkhead transcription factor (termed Mac-1-regulated forkhead [MFH] and found subsequently to be identical to Foxp1) that is downregulated after Mac-1 clustering and controls monocyte differentiation as a transcriptional repressor of the $c$-fms promoter.

\section{Results}

Mac-1 clustering and gene expression. To identify novel genes regulated by the clustering and activation of $\mathrm{Mac}-1$, we carried out DD-PCR using total RNA extracted from Mac-1-clustered and -nonclustered THP-1 cells. We clustered Mac-1 by inducing adhesion of THP-1 cells to immobilized fibrinogen, as previously reported (12). When THP-1 cells were stimulated with the $\beta_{2}$-integrin-activating mAb KIM127 (15), they adhered robustly to wells coated with fibrinogen and blocked with gelatin, but not to wells coated with gelatin alone (Figure 1A). Adhesion of the cells to fibrinogen was inhibited by LPM19c (95\% $\pm 5 \%$ inhibition), a mAb that binds to the $\alpha_{\mathrm{M}} \mathrm{I}$ domain of Mac- 1 and blocks ligand binding (16), indicating that, under these experimental conditions, THP-1 cell adhesion to fibrinogen is Mac-1-dependent.

Mac-1-dependent gene expression was examined using DD-PCR with RNA from adherent (i.e., Mac-1-clustered) THP-1 cells and from cells treated identically except that adhesion was inhibited by LPM19c (i.e., Mac-1-nonclustered). We analyzed cDNA expression patterns from three independent clustering experiments, limiting our focus to genes with qualitatively similar changes in all three experiments. By comparing the intensity of DD-PCR bands, we observed that clustering of Mac- 1 is accompanied by changes in gene expression. Figure $1 \mathrm{~B}$ illustrates a band with reduced intensity after clustering in all three experiments. A cDNA fragment, termed $12 \mathrm{CC} 4$ based on the primer pair combination, was recovered from the DD-PCR gel. Northern blot analysis (Figure 1C) using this 284bp labeled probe detected a $4.9-\mathrm{kb}$ band that was downregulated in mRNA purified from Mac-1-clustered THP-1 cells compared with mRNA purified from Mac-1-nonclustered THP-1 cells.

We isolated the full-length cDNA of this gene by a combination of $5^{\prime}$ and $3^{\prime}$ rapid amplification of cDNA ends (RACE). The full-length cDNA sequence of $12 \mathrm{CC} 4$ consisted of $4,954 \mathrm{bp}$, encoding a predicted protein consisting of 677 amino acids (assigned GenBank accession number AF250920 on March 30, 2000). In vitro-coupled transcription and translation of native full-length $12 \mathrm{CC} 4$ produced a single band of predicted size (molecular weight 285,000 ) (Figure 1D).

12CC4 contains a winged-helix DNA-binding domain (amino acids 465-536), which defines the protein as likely belonging to the hepatic nuclear factor-3/forkhead domain family of wingedhelix transcription factors (17). Members of this family take part in a wide range of normal development events including the control of cellular differentiation and proliferation, pattern formation, and signal transduction (18). On the basis of this homology, we renamed $12 \mathrm{CC} 4$ as MFH. Since we deposited the MFH sequence, one independent group cloned the identical sequence using an expression-cloning strategy to identify an antigen for a novel mAb that recognized a nuclear protein in normal tonsil and was also overexpressed in a subset of diffuse large B cell lymphomas (19). Based on its forkhead homology, this protein was assigned a putative forkhead designation as Foxp1. Using a degenerate-cloning strategy to identify novel forkhead family members involved in lung development, a second independent group has cloned the likely mouse homolog of Foxp1, nearly identical in sequence to human $M F H /$ Foxp1 except for an expanded glutamine-rich region (amino acids 55-230) resulting in a 705-amino acid rather than a 677-amino acid protein (20). Foxp1 functions as a transcriptional repressor of the surfactant protein $C$ and CC10, two genes potentially important in the developing airway epithelium. Based on the amino acid identity of 12CC4 and human Foxp1, we will refer to $12 \mathrm{CC} 4$ as $M F H / F o x p 1$.

$M F H / F o x p 1$ expression and monocyte differentiation. Since Mac-1 clustering downregulates $M F H /$ Foxp 1 expression and since Mac- 1 is required for the firm adhesion of monocytes and their transmigration into tissues, we became intrigued by the possibility that MFH/ Foxp1 may play a role in monocyte differentiation. We first analyzed

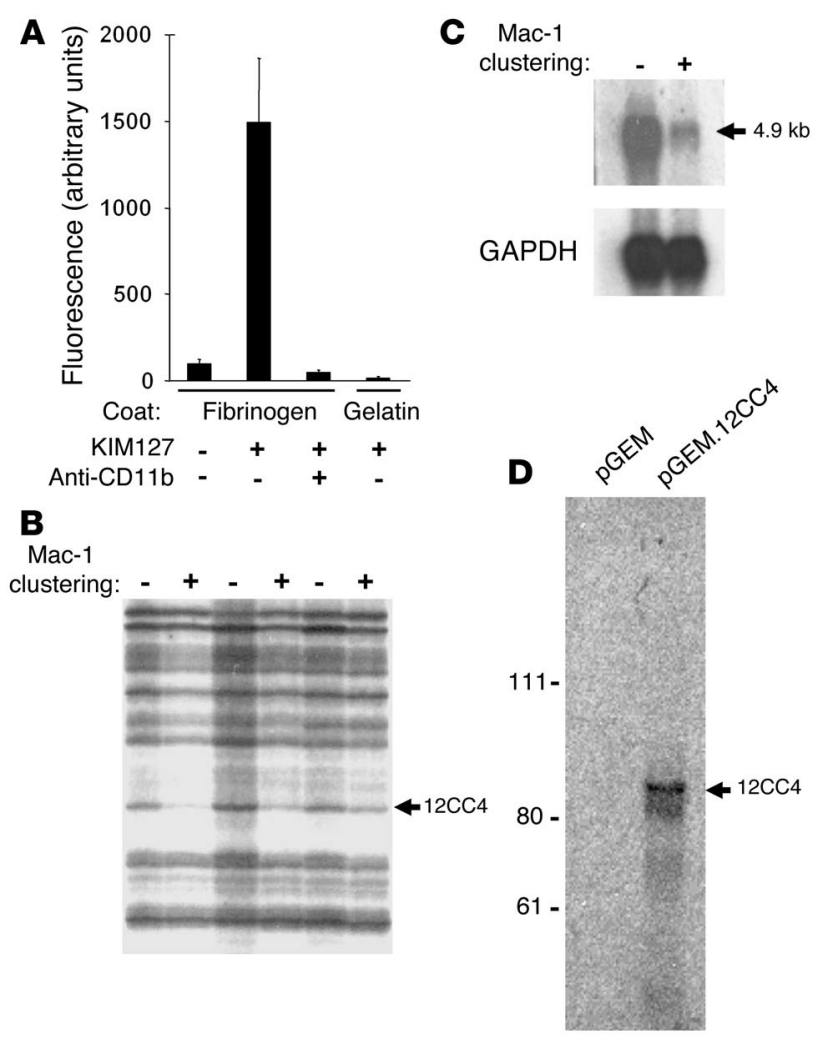

Figure 1

Clustering of Mac-1 downregulates expression of 12CC4/MFH/Foxp1. (A) Clustering of Mac-1. Cytokine-treated THP-1 cells were added to fibrinogen-coated and gelatin-blocked wells. Adhesion was promoted by the addition of the anti-CD18 stimulating mAb KIM127 $(5 \mu \mathrm{g} / \mathrm{ml})$ in the presence and absence of the anti-CD11b mAb LPM19c $(10 \mu \mathrm{g} / \mathrm{ml})$. After washing, adhesion was quantified by measurement of the fluorescence of 2',7'-bis-(2-carboyethyl)-5-(and-6)-carboxyfluorescein, acetoxymethyl ester-loaded (BCECF AM-loaded) THP-1 cells (Molecular Probes, Eugene, Oregon, USA). Triplicate determination (mean \pm SD) representative of three separate experiments is shown. (B) Clustering of Mac-1 downregulates expression of 12CC4. Mac-1-dependent gene expression was examined using DD-PCR with RNA from Mac1-clustered (+) and -nonclustered (-) THP-1 cells. cDNA expression patterns were analyzed from three independent clustering experiments. The arrow designates a band (12CC4) with reduced intensity after clustering in all three experiments. (C) Northern analysis. Northern blots containing $1 \mu \mathrm{g} / \mathrm{lane}$ of mRNA isolated from Mac-1-clustered and -nonclustered THP-1 cells were probed with ${ }^{32 P}$-labeled 12CC4 cDNA. RNA size is indicated in kilobases. (D) In vitro transcription and translation of vector alone and 12CC4. In vitro-coupled transcription and translation of native full-length $12 \mathrm{CC} 4(4,954 \mathrm{bp})$ produced a single band of predicted size (molecular weight $\sim 85,000$ ). 
A

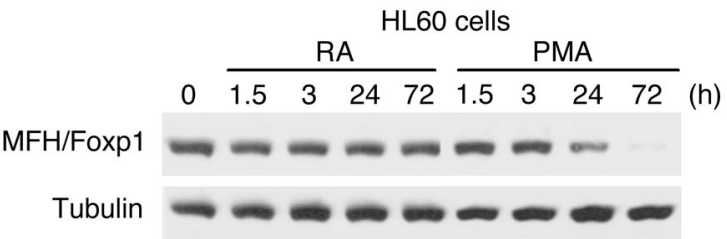

B

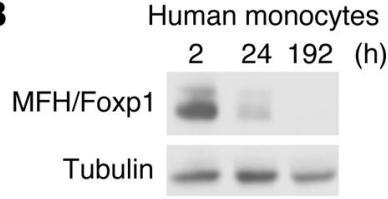

\section{Figure 2}

Monocyte differentiation and expression of MFH/Foxp1. (A) HL60 cells. The expression of MFH/Foxp1 was examined in an in vitro system of myelomonocytic differentiation. HL60 cells were treated with PMA (10 nM) to induce monocytic differentiation (42) or with RA (100 nM) to induce granulocytic differentiation (22). (B) Human monocytes. MFH/Foxp1 expression was also assessed after adhesion-induced differentiation of human peripheral blood monocytes. At the indicated times, cells were lysed in situ with SDS-PAGE reducedsample buffer and then immunoblotted sequentially with anti-MFH/ Foxp1 and anti-tubulin antibodies.

MFH/Foxp1 expression in an in vitro system of myelomonocytic differentiation. HL60 cells can differentiate along both the monocytic and the granulocytic lineages. While PMA induces monocyte differentiation (21), all-trans retinoic acid (RA) stimulates granulocytic differentiation (22). MFH/Foxp1 is expressed in untreated HL60 cells, and its expression was markedly reduced during PMA-induced monocyte, but not RA-induced granulocyte, differentiation (Figure 2A). We also examined $M F H / F o x p 1$ expression after adhesioninduced differentiation of human peripheral blood monocytes. Macrophage transformation of these cells was also associated with markedly reduced expression of $M F H / F o x p 1$ (Figure 2B).

$M F H / F o x p 1$ modulates monocyte differentiation. In order to study a role for $M F H / F o x p 1$ in monocyte differentiation, we performed experiments examining the effects of overexpression of $M F H / F o x p 1$ on PMA-induced monocyte differentiation in vitro. As read-outs, we examined cellular morphology, adhesion molecule expression and function, and phagocytosis. We overexpressed $M F H / F o x p 1$ by two different strategies. The first strategy involved stably transfecting HL60 cells using a pcDNA3.1/myc-his(-)A vector containing full-length $M F H / F o x p 1$. High-expressing $M F H / F o x p 1$ mixed clones were isolated by G418 selection and single clones by limiting-dilution techniques. Overexpression of MFH/Foxp1-myc (Figure $3 \mathrm{~A}$ ) prevented PMA-induced cell spreading and morphologic macrophage-like differentiation (Figure $3 \mathrm{~B}$ ).

The second strategy used murine stem cell virus (MSCV) retroviral constructs harboring MFH/Foxp1-FLAG and HL60 cells that had been engineered for retroviral transduction. HL60 cells were transduced with MSCV.MFH/Foxp1.GFP or vector control (MSCV. GFP), and the effect of overexpression of $M F H / F o x p 1$ on CD11b expression and function was assessed. Retroviral transduction was efficient, and after cell sorting, more than $90 \%$ of cells were GFP-positive. Overexpression of $M F H / F o x p 1$ markedly attenuated PMA-induced CD11b expression over time (Figure 4A), resulting in impaired CD11b-dependent adhesion to fibrinogen (Figure 4B). We also tested the effect of $M F H / F o x p 1$ on phagocytic activity, the longest-known function of macrophages. HL60 cells transduced with control virus (MSCV.GFP) and stimulated with PMA were incubated with fluorescent zymosan particles for 2 hours. As shown in Figure 4C, control virus-transduced cells robustly took up zymosan particles and became intensely red-fluorescent. In contrast, cells transduced with MSCV.MFH/Foxp1.GFP took up fewer zymosan particles $(60 \pm 7$ beads per low-power field vs. $180 \pm 14$ beads per field, $n=3-4, P<0.01$ ), indicating that they had significantly reduced phagocytic activity. Taken together, these results demonstrate that expression of $M F H /$ Foxp 1 is capable of inhibiting monocyte differentiation in vitro.

Regulation of c-fms expression by MFH/Foxp1. We next tested whether $M F H / F o x p 1$ could regulate expression of $c$-fms and PU.1, two genes known to participate in monocyte differentiation and whose deficiency is associated with a paucity of tissue macrophages. HL60 cells transduced with control virus (MSCV.GFP) were stimulated with PMA, and the expression of $c$-fms and PU.1 was assessed by Northern analysis. As shown in Figure 5A, PMA induced the expression of these macrophage-specific genes in control virus-transduced cells. In contrast, cells transduced with MSCV.MFH/Foxp1.GFP demonstrated delayed and attenuated expression of $c$-fms. Overexpression of $M F H / F o x p 1$ had no effect on the expression of PU.1.

Since Mac-1 clustering downregulated the expression of $M F H /$ Foxp1 and overexpression of $M F H / F o x p 1$ attenuated the expression of $c$-fms, we hypothesized that Mac-1 engagement would modulate $c$-fms expression. Indeed, clustering of Mac- 1 decreased the expression of $M F H / F o x p 1$ and increased the expression of c-fms mRNA (Figure 5B) in THP-1 cells. Western analysis con-

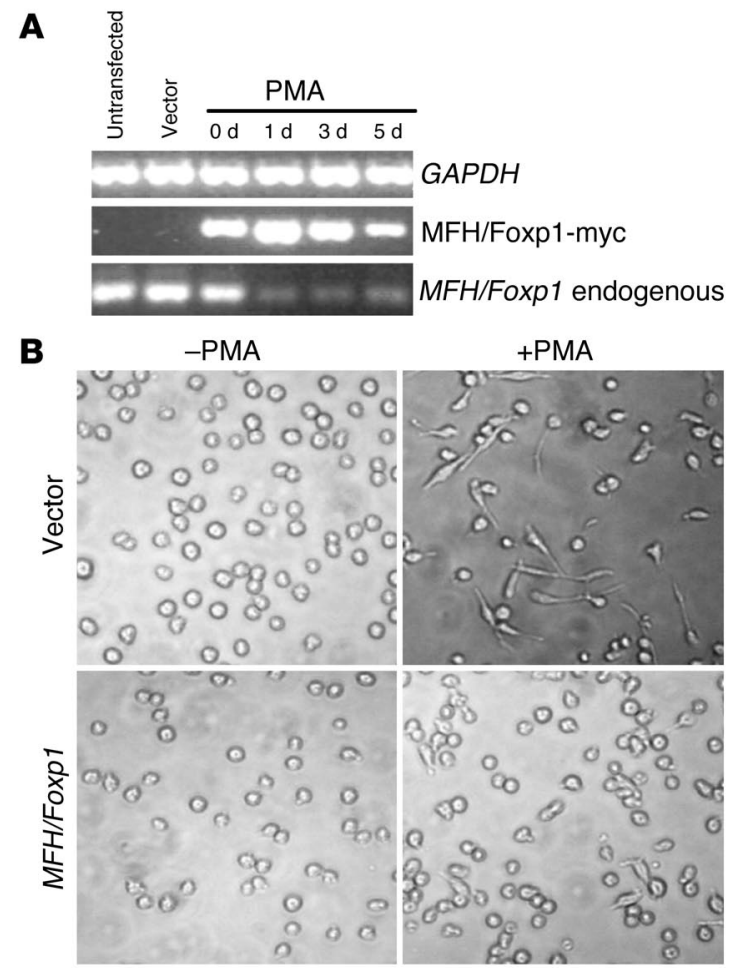

Figure 3

Effect of overexpression of MFH on PMA-induced monocyte differentiation of HL60 cells. HL60 cells were stably transfected using pcDNA3.1/myc-his(-)A vector containing full-length MFH/Foxp1. (A) Semiquantitative PCR of endogenous MFH/Foxp1 and MFH/Foxp1myc. (B) Light microscopy 12 hours after PMA treatment of HL60 cells. 


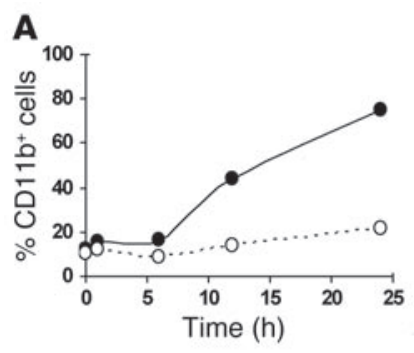

B
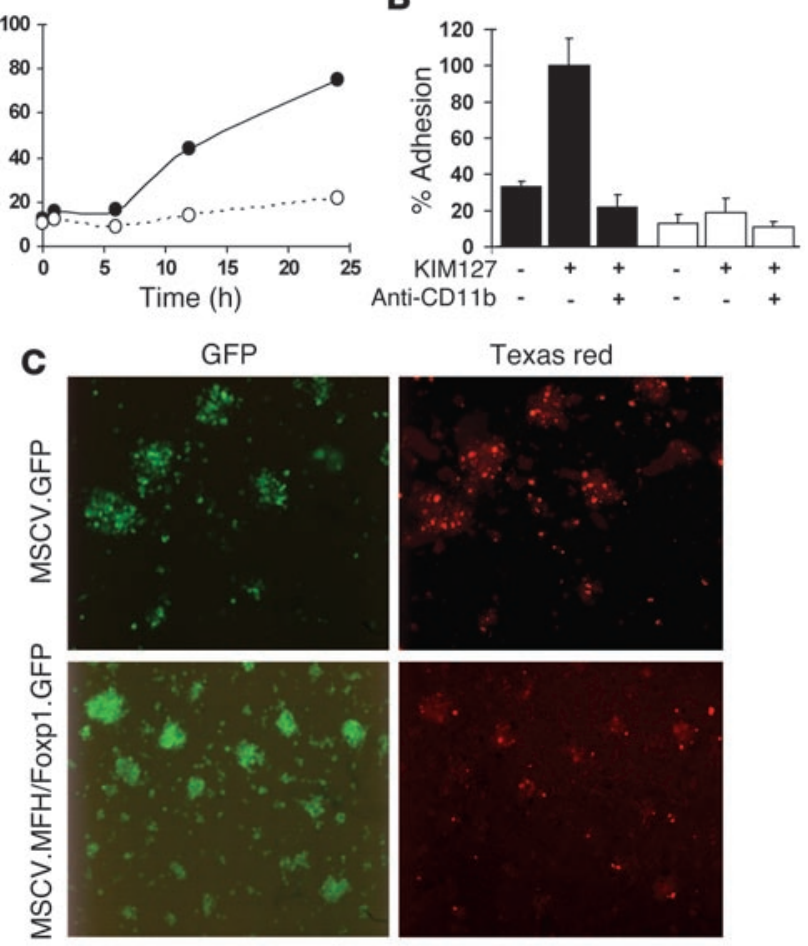

firmed that the Mac-1-dependent changes in $M F H / F o x p 1$ and $c$-fms expression also occurred at the protein level (Figure 5C, left panel). Importantly, we observed a similar expression pattern of $M F H / F o x p 1$ and $c$-fms after Mac-1 clustering in peripheral blood human monocytes (Figure 5D).

Given our prior report that clustering of Mac-1 under identical experimental conditions recruits Toll/IL-1 receptor signaling intermediates to modulate NF- $\kappa B$ activity (12), we were intrigued by the possibility that NF- $\mathrm{KB}$ may play an upstream role in regulating $M F H / F o x p 1$ and $c$-fms. Tyrosine kinases (23) and MAPK (9) are also known to be activated after Mac-1 engagement. Therefore, to gain insight into whether these signaling pathways are involved in regulating $M F H / F o x p 1$ and $c-f m s$, we examined the effect of a panel of inhibitors on $M F H / F o x p 1$ and $c$-fms expression in response to Mac-1 clustering (Figure 5C, right panel). Inhibition of PI3K (wortmannin) did not affect $M F H / F o x p 1$ and $c$-fms expression. In contrast, inhibition of NF- $\mathrm{KB}$ activation with the proteosome inhibitor bortezomib/PS-341 (24) had a striking effect on the expression of $M F H / F o x p 1$ and $c$-fms in response to Mac-1 clustering. Bortezomib enhanced, rather than decreased, $M F H / F o x p 1$ expression and abolished the expression of c-fms. Inhibition of p38/SAPK2 MAPK (SB203580) or tyrosine kinases (herbimycin A) attenuated the downregulation of $M F H / F o x p 1$ and the upregulation of $c-f m s$ in response to Mac-1 clustering. Taken together, these observations suggest that, although multiple signaling pathways may be involved, NF- $\kappa \mathrm{B}$ likely plays an important role in the regulation of $M F H / F o x p 1$ and $c$-fms.

$M F H / F o x p 1$ functions as a transcriptional repressor of $c$-fms. We next tested whether $M F H / F o x p 1$ could regulate expression of the $c$-fms promoter. Examination of the proximal region of the human $c$-fms promoter revealed the presence of multiple Foxp1-like (25) binding sites. Cotransfection studies showed that full-length $M F H / F o x p 1$ decreased $c$-fms promoter activity by $72 \% \pm 11 \%(P<0.01)$ (Fig-

\section{Figure 4}

Effect of overexpression of MFH on CD11b expression, cellular adhesion, and phagocytosis. (A) CD11b expression. HL60 cells, modified for MSCV retroviral transduction, were transduced with MSCV.MFH/ Foxp1.GFP (open circles) or vector control (MSCV.GFP) (filled circles). Transduced HL60 cells were then treated with PMA (10 nM), and CD11b expression was assessed over time by FACS analysis using r-PE-conjugated anti-CD11b mAb (LPM19c). Data represent percentage CD11b-positive cells over time from three independent experiments. (B) Adhesion. Transduced HL60 cells (MSCV.GFP, black bars; MSCV.MFH/Foxp1.GFP, white bars) were harvested 24 hours after PMA treatment to investigate their adhesion to fibrinogen-coated microtiter wells. Adhesion was induced by the addition of KIM127. CD11b-dependence was examined by incubation of cells with the antiCD11b mAb (LPM19c). Data represent percentage adhesion of vector control HL60 cells treated with the $\beta_{2}$-integrin-stimulating mAb KIM127 (mean $\pm \mathrm{SD}, n=3$ ). (C) Phagocytosis. GFP-expressing HL60 cells were cultured in the presence of Texas red-conjugated zymosan particles for 2 hours at $37^{\circ} \mathrm{C}$ as described in Methods. Cells were viewed for internalization of the particles by fluorescence microscopy after quenching of extracellular fluorescence with trypan blue. Assays were performed in triplicate from at least three independent experiments.

ure 6A). In contrast, expression of $M F H /$ Foxp1 did not affect the promoter activity of macrophage migration inhibitory factor or PPAR $\gamma$-1 reporter constructs (data not shown) or the expression of $\beta$-galactosidase from the pCMV- $\beta$-gal reference plasmid; this indicates that the observed repression of $c$-fms is likely specific.

Identification of repression domain. Recent studies have reported that an independent transcription-repressor domain of murine Foxp1 and Foxp2, which are highly homologous to human MFH/ Foxp1, lies in the $\mathrm{N}$-terminal end of the protein spanning the zincfinger and leucine-zipper domains (20). However, human MFH/ Foxp1 is a smaller protein with a contracted polyglutamine region, which raises the possibility that an alternative mechanism(s) may be responsible for human MFH/Foxp1-mediated transcriptional repression. To characterize the region(s) within $\mathrm{MFH} / \mathrm{Foxp} 1$ that is responsible for the transcriptional repression activity observed on the $c$-fms promoter, a series of $\mathrm{N}$ - and C-terminal MFH/Foxp1 deletion mutants were generated, cotransfected with a $c$-fms reporter construct, and assayed for loss of repression in transient transfection assays (Figure 6A). These deletion mutants were designed on the basis of the predicted modular domain structure of MFH/Foxp1 in order to isolate the potential contributions of the glutamine-rich region (amino acids 55-200), the zinc-finger region (amino acids 308-331), and the winged-helix/forkhead DNA-binding region (amino acids 465-536) to the repressor activity of MFH/Foxp1. In all cases the authenticity of each construct was confirmed by sequence analysis and immunoblotting using anti-MFH/Foxp1 and anti-c-myc antibodies (Figure 6B). N-terminal deletion analysis demonstrated that repression of the c-fms promoter required the glutamine-rich domain (Figure 6A). Deletion of the winged-helix/forkhead DNA-binding domain was also accompanied by loss of repression. Taken together, these results suggest that both the glutamine-rich and the winged-helix/ forkhead DNA-binding domains of MFH/Foxp1 are required for transcriptional repression.

$M F H / F o x p 1$ represses transcription through binding sites within the c-fms promoter. Given that MFH/Foxp1 repression required the DNAbinding domain, we next sought to determine the region within the $c$-fms promoter (2) that mediated this effect. Examination of the 

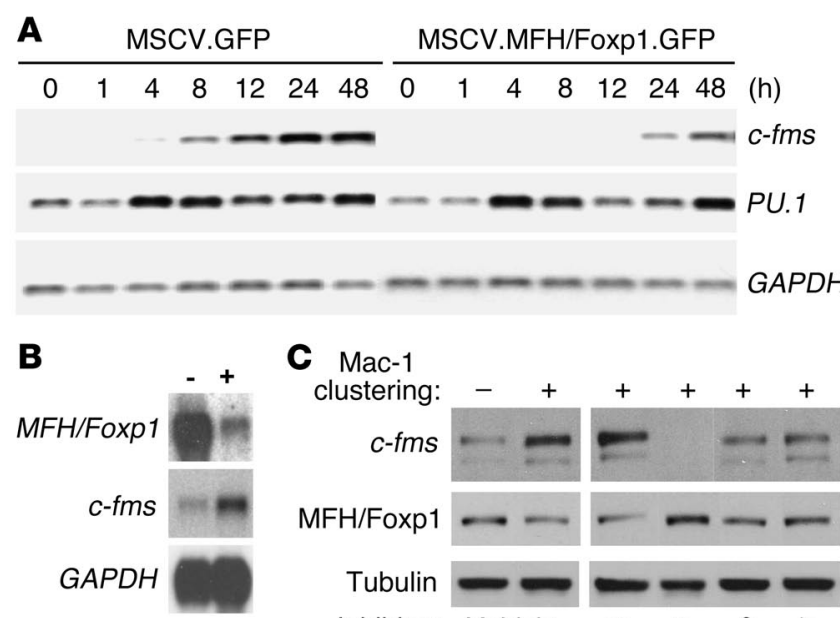

\section{Mac-1}

clustering:

$$
\text { c-fms }
$$
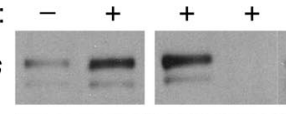

$+\quad+$
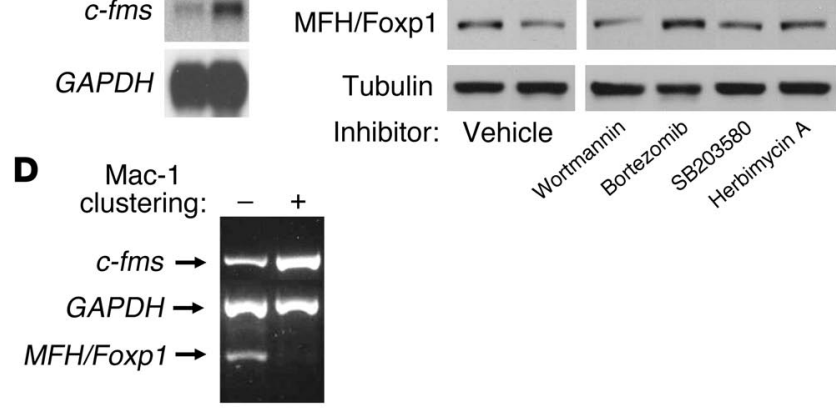

Tubulin

Inhibitor: Vehicle

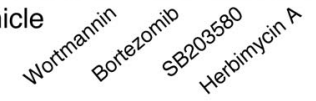

c-fms promoter revealed three uncharacterized sequences (Figure 7A), that can be aligned with a Foxp1-like (25) consensus sequence (Figure 7B). In the first set of electrophoretic mobility shift assay (EMSA) experiments, we focused on the -115 CAAGGCTTTGTTTTCTTCTTAA -94 site, designated binding site-1 wild-type (bs1WT), in which the Foxp1-binding site is underlined, and observed a specific interaction between bs1WT and GST-MFH/ Foxp1 (Figure 7B). Mutation of several bases within this sequence

\section{Figure 5}

MFH/Foxp1 and c-fms expression. (A) Overexpression of MFH/Foxp1 attenuates expression of $c-f m s$. HL60 cells transduced with control virus (MSCV.GFP) or MSCV.MFH/Foxp1.GFP were stimulated with PMA (10 nM), and the expression of $c$-fms and PU.1 over time was assessed by Northern analysis. (B) Clustering of Mac-1 enhances c-fms expression. Northern blots containing $1 \mu \mathrm{g} / \mathrm{lane}$ of mRNA isolated from Mac-1-clustered (+) and -nonclustered (-) THP-1 cells were probed with ${ }^{32} \mathrm{P}$-labeled $\mathrm{c}$-fms and MFH/Foxp $1 \mathrm{cDNA}$ probes. (C) Regulation of MFH/Foxp1 and c-fms protein by Mac-1. MFH/Foxp1 and $c$-fms proteins were assessed after Mac- 1 clustering. THP-1 cells were lysed 24 hours after clustering with SDS-PAGE reduced-sample buffer and then immunoblotted sequentially with anti-MFH/Foxp1, anti-c-fms (130 kDA and $150 \mathrm{kDa}$ ), and anti-tubulin antibodies. Signaling inhibitors included $250 \mathrm{nmol} / \mathrm{l}$ wortmannin, $5 \mu \mathrm{mol} / \mathrm{l}$ bortezomib, $100 \mathrm{nmol} / \mathrm{l} \mathrm{SB203580}$, and $1 \mu \mathrm{mol} / \mathrm{l}$ herbimycin A. (D) MFH/Foxp1 in human monocytes. Semiquantitative PCR of MFH/Foxp1 and c-fms from RNA isolated from Mac-1-clustered (+) and -nonclustered (-) peripheral blood human monocytes.

(i.e., bs1mut1, bs1mut2, bs1mut3) that are conserved in the Foxp1 consensus reduced the binding of GST-MFH/Foxp1 (Figure 7B). The ability of MFH/Foxp1 to interact with the two additional forkhead-like sites within -13 GGAAAACAAGACAAACAGCC +7 was examined by synthesis of a single oligomer, designated bs $2 /$ $3 \mathrm{WT}$. We observed a specific interaction between bs $2 / 3 \mathrm{WT}$ and GST-MFH/Foxp1, and mutation of this sequence (bs2/3mut: -13 GGAtgcagAGACgtcgtGCC +7; mutated bases in lower case) eliminated binding (Figure 7B). Taken together, these observations suggest that MFH/Foxp1 is capable of interacting with forkhead-like sites within the $c$-fms promoter.

The monocyte-specific expression of $c$-fms is regulated by at least three transcription factors interacting with critical regions of the c-fms promoter, including PU.1, AML1, and C/EBP $\alpha$ (2). Since
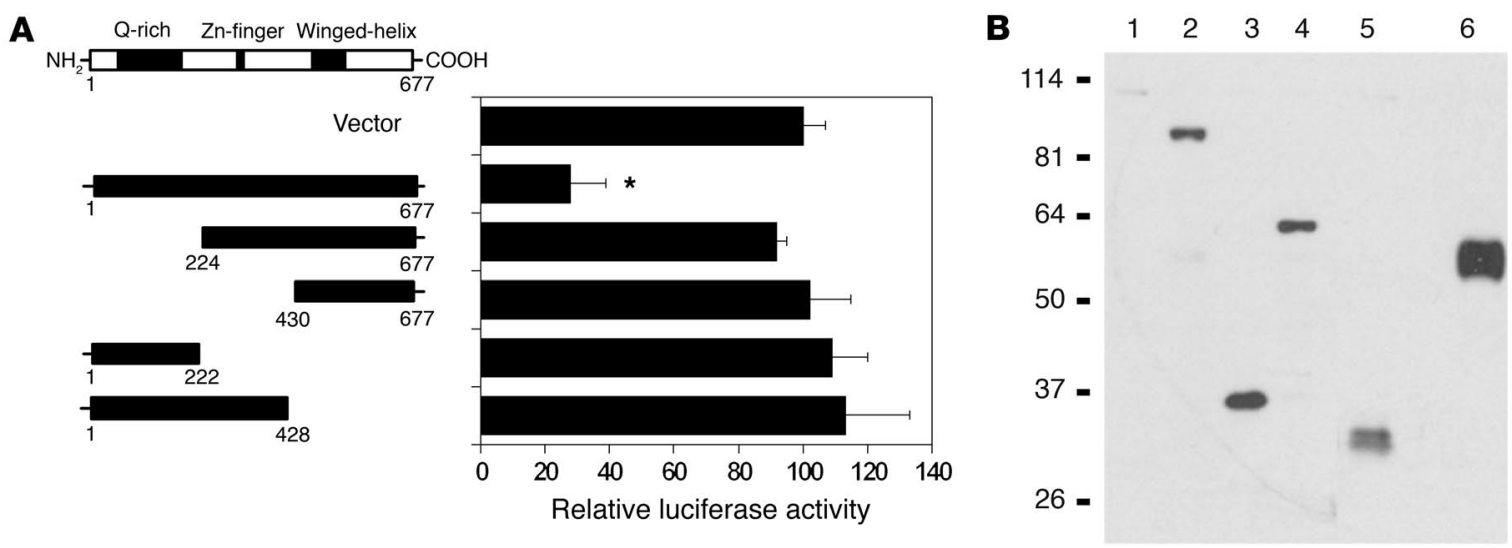

\section{Figure 6}

Effect of full-length MFH/Foxp1 and amino- and carboxy-terminal deletion mutants on c-fms promoter activity. (A) Activity of the $c$-fms promoter. To map the repression domain of MFH/Foxp1, a series of $\mathrm{NH}_{2}$ - and $\mathrm{COOH}$-terminal MFH/Foxp1 deletion mutants were generated. Deletion mutants were designed on the basis of the predicted modular domain structure of MFH/Foxp1 in order to establish the contribution of the glutamine-rich (Q-rich, amino acids 55-200), zinc-finger (Zn-finger, amino acids 308-331), and winged-helix/forkhead DNA-binding (amino acids 465-536) regions to the repressor activity of MFH/Foxp1. NIH 3T3 cells were transfected with $0.5 \mu \mathrm{g} c$-fms reporter gene plasmid, $0.05 \mu \mathrm{g}$ pCMV- $\beta$-gal, and expression plasmids $(5.5 \mu \mathrm{g})$ for pcDNA3.1 (vector), full-length MFH/Foxp1, or deletion mutants added to each well of a 12well plate. Luciferase activities were determined, normalized on the basis of $\beta$-galactosidase activity, and plotted as percent promoter activity compared with that induced by treatment with vector alone. Triplicate determination of three to five independent experiments (mean \pm SD) is shown. ${ }^{*} P<0.01$. (B) Verification of MFH/Foxp1 mutant expression. Western blot analysis of NIH 3T3 lysates verifies expression of MFH/Foxp1 and $\mathrm{NH}_{2}$ - and $\mathrm{COOH}$-terminal MFH/Foxp1 deletion mutants: vector alone (lane 1), full-length MFH/Foxp1 amino acids 1-677 (lane 2), $430-677$ (lane 3), 224-677 (lane 4), 1-222 (lane 5), and 1-428 (lane 6). 
the MFH/Foxp1 binding sites are adjacent to the C/EBP $\alpha(-84$ ATTTCCAAAC -75) and PU.1 (-51 AAAGGGGAAGAAGAGGATCA -32) binding sites, we examined the effect of MFH/Foxp1 on PU.1- and C/EBP $\alpha$-stimulated $c$-fms promoter activity. HeLa cells were cotransfected with PU.1 and/or C/EBP $\alpha$ expression construct along with a luciferase reporter carrying the $c$-fms promoter region spanning the Foxp1 and C/EBP sites. Transfection of PU.1 stimulated $c$-fms luciferase activity two- to threefold, C/EBP $\alpha$ stimulated $c$-fms luciferase activity tenfold, and the combination of PU.1 and $\mathrm{C} / \mathrm{EBP} \alpha$ stimulated luciferase activity 14 -fold compared with vector alone (Figure $7 \mathrm{C}$ ). Cotransfection of $M F H /$ Foxp 1 with PU.1 and $\mathrm{C} / \mathrm{EBP} \alpha$ significantly attenuated PU.1-stimulated, C/EBP $\alpha$-stimulated, and combined PU.1/C/EBP $\alpha$-stimulated $c$-fms promoter activity $(62 \%, 82 \%$, and $61 \%$ inhibition, respectively).

Next, we examined the effect of mutations of the $c$-fms promoter that eliminated MFH/Foxp1 binding in EMSAs (Figure 7B). Mutation of bs $1 \mathrm{WT}$ and bs $2 / 3 \mathrm{WT}$ sites (i.e., bs $1 / \mathrm{bs} 2 / 3$ triple mutant) attenuated MFH/Foxp1-mediated repression of the $c$-fms promoter, with complete elimination of repression of combined PU.1/C/EBP $\alpha-$ stimulated $c$-fms promoter activity (Figure 7C). Taken together, these observations indicate that MFH/Foxp1 binds specifically to heretofore uncharacterized Foxp1 forkhead sites within the $c$-fms promoter and operates as a transcriptional repressor of $c$-fms expression.

Mac-1 deficiency and MFH/Foxp1. We hypothesized that deficiency of Mac-1 would lead to impaired downregulation of $M F H / F o x p 1$ expression and, in turn, altered monocyte maturation in vivo. To explore signaling functions of Mac-1 distinct from its role in migration, we selected the thioglycollate peritonitis model, because inflammatory cell recruitment is unaffected by Mac-1 deficiency $(26,27)$. Peritoneal cells were harvested 16 hours after thioglycollate instillation, and $M F H /$ Foxp 1 expression was assessed by immunoblotting. $M F H /$ Foxp 1 expression was markedly reduced in wild-type compared with Mac-1-deficient cells (Figure 8), suggesting that Mac-1 regulates $M F H /$ Foxp1 expression in vivo.

To determine whether Mac-1 signaling modulates monocyte differentiation in vivo, we performed flow cytometric analysis of thioglycollate-induced peritoneal inflammatory cells in wild-type and Mac-1-deficient mice using mAb's, including ER-MP12 and ER-MP20, that recognize antigens associated with distinct stages of increasing monocyte maturation (28). ER-MP12 recognizes the CD31 antigen (PECAM-1) (29), while ER-MP20 is directed to the

\section{Figure 7}

$\mathrm{MFH} /$ Foxp1 represses transcription of $c$-fms through Foxp1-like binding sites within the $c$-fms promoter. (A) Examination of the $c$-fms promoter revealed three uncharacterized sequences (bs1, bs2, and bs3) that can be aligned with a Foxp1-like (25) consensus sequence. (B) EMSA was performed as described in Methods. The GST-MFH/Foxp1 fusion protein was incubated with ${ }^{32} \mathrm{P}$-labeled bs1WT, bs2/3WT, or mutant $c$-fms duplex oligonucleotides. A single dominant DNA-protein complex was seen with both bs1WT and bs2/3WT (arrows). (C) Effect of $M F H / F o x p 1$ on wild-type and mutant $c$-fms promoter activity stimulated by PU. 1 and C/EBP $\alpha$. HeLa cells were cotransfected with PU.1, C/EBP $\alpha$, and/or MFH/Foxp1 expression constructs along with a wild-type $c$-fms luciferase reporter (black bars) or bs $1 / \mathrm{bs} 2 / 3$ triple mutant $c$-fms luciferase reporter (white bars). This region of the $c$-fms promoter $(-175$ to +71$)$ contains the transcription factor binding sites for C/EBP $\alpha$ and PU.1. Luciferase activities were determined, normalized on the basis of $\beta$-galactosidase activity, and plotted as percent promoter activity compared with that of the wild-type $c$-fms promoter treated with vector alone (mean $\pm \mathrm{SD}, n=3$ ).
Ly-6C antigen (30). Monocyte maturation is accompanied by a shift from ER-MP12 $2^{\text {high }} /$ ER-MP20 $0^{\text {low }}$ to ER-MP12 $2^{+}$ER-MP20 $0^{+}$and ERMP12 low/ER-MP20 high populations of cells. Mature macrophages no longer express ER-MP12 or ER-MP20 (31). Although ER-MP12 and ER-MP20 recognize other cells, double staining with MOMA-2, which recognizes both bone marrow monocyte/macrophage precursors and mature macrophages (32), permits the definitive identification of cells of the monocytic lineage.

\section{A -115 CAAGGCTTTGTTTTCTTCTTAA -94, bs1 and -13 GGAAAACAAGACAAACAGCC +7 \\ B \\ Foxp1-binding sequence TT ATT T \\ Promoter bs1WT -115 C A A G G C T T T G T T T T CT T C T T A A -94 bs1

$\begin{array}{ll}\text { Promoter_bs1mut1 } & \text { CAAGGCTggaTTTTCTTCTTAA } \\ \text { Promoter_bs1mut2 } & \text { CAAGGCTTTGagaTCTTCTTAA } \\ \text { Promoter_bs1mut3 } & \text { CAAGGCTTTGTCCgTTCTTAA }\end{array}$ \\ Promoter_bs $2 / 3 W T-13$ G G A A A A C A A G A C A A A C A G C C +7 bs2 bs 3 \\ Promoter_bs2/3mut GGAtg c g A GACg t c g t GCC}

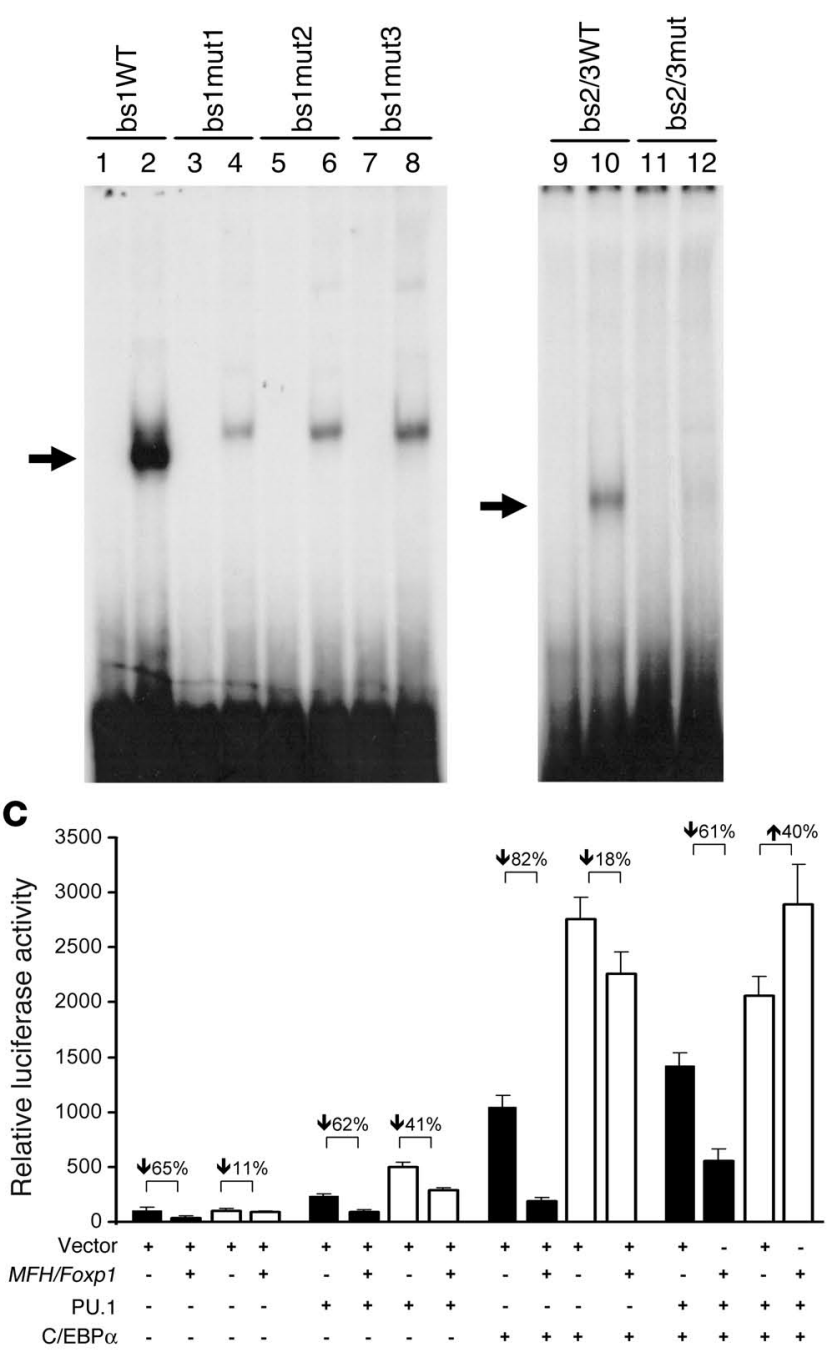




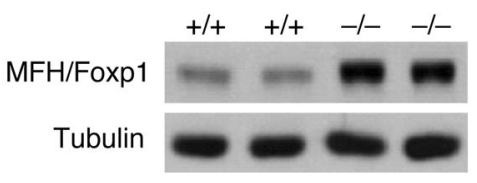

Figure 8

Mac-1 deficiency and MFH/Foxp1. Peritoneal cells were harvested from two wild-type (+/+) and two Mac-1-deficient (-/-) mice 16 hours after thioglycollate instillation, and cells were lysed with SDS-PAGE reduced-sample buffer and then immunoblotted sequentially with anti$\mathrm{MFH} /$ Foxp1 and anti-tubulin antibodies.

Peritoneal inflammatory cells were isolated and double-stained with MOMA-2 and either ER-MP12 or ER-MP20 and then analyzed by flow cytometry (Figure 9; Table 1). ER-MP20 staining was significantly reduced in MOMA-2 $/$ Mac-1-deficient compared with wild-type cells. There was a nonsignificant increase in ERMP12 staining in MOMA-2+/Mac-1-deficient cells. Alteration in monocyte maturation was also confirmed by reduced staining of the myeloid marker Gr-1 (33) in MOMA-2+/Mac-1-deficient compared with wild-type cells. Importantly, diminished MP-20 and Gr-1 staining in Mac-1-deficient MOMA-2 $2^{+}$cells is similar to the pattern of staining in PU.1-deficient MOMA- $2^{+}$cells (28). Taken together, these observations suggest that Mac-1 signaling modulates monocyte maturation in vivo.

\section{Discussion}

The precise external signals that control differentiation of peripheral blood monocytes to tissue macrophages are incompletely defined. In this study, we have shown that Mac-1 engagement downregulates the expression of $M F H / F o x p 1$, a transcriptional repressor of $c$-fms. Furthermore, deficiency of Mac-1 is associated with altered regulation of $M F H / F o x p 1$ and monocyte maturation in vivo. This represents a new pathway for integrin-dependent modulation of gene expression and control of cellular differentiation.

Integrins regulate gene expression through the activation of transcription factors. A direct link between $\beta_{2}$-integrins and transcription factors has remained elusive until the recent demonstration that the cytoplasmic tail of LFA-1 interacts with the transcriptional coactivator JAB1 and modulates AP-1 activity by regulating JAB1 nuclear localization (11). Given our prior observation that Mac-1 associates with IL-1 receptor-associated kinase 1 (IRAK1) and modulates NF- $\kappa \mathrm{B}$ activity in a cascade involving TNF receptor-associated factor 6 (TRAF6) and TGF- $\beta$-activated kinase 1 (TAK1) (12), we were intrigued by the possibility that NF-кB may play an upstream role in regulating $M F H / F o x p 1$ and $c$-fms. Inhibi-

\section{Figure 9}

Mac-1 deficiency and monocyte maturation. Peritoneal cells from wild-type (WT) and Mac-1-deficient $\left(\mathrm{MaC}^{-1^{-/-}}\right)$mice were harvested and double-stained with FITC-conjugated MOMA-2, biotinylated ERMP12 or ER-MP20, and PE-conjugated Gr-1 antibodies. ER-MP12 and ER-MP20 were stained using streptavidin-PE-cyanine5. MOMA-2 versus ER-MP12, ER-MP20, or Gr-1 FACS data of wild-type and Mac-1 $1^{-1-}$ cells demonstrate single-positive, double-positive, and unstained cells. The quadrants were determined based on the background staining of cells with isotype control antibodies. Values represent mean fluorescence intensity (MFI) found in gated wild-type and Mac-1-deficient MOMA-2+ population. tion of NF-кB activation with the proteosome inhibitor bortezomib (24) had a striking effect on the expression of MFH/Foxp1 and $c$-fms, increasing rather than decreasing $M F H / F o x p 1$ expression and blocking $c$-fms expression in response to Mac-1 clustering. However, since bortezomib has other biological actions, including the inhibition of IL-6-triggered MAPK activation (24), this finding does not rule out a role for other signaling intermediates. Identification of the signaling pathways linking Mac-1 clustering and $M F H / F o x p 1$ will require detailed characterization of the $M F H / F o x p 1$ promoter region and are the focus of ongoing studies. Emerging experimental evidence suggests that NF- $\mathrm{KB}$ is capable of gene trans-repression (34-36) and provides an important line of mechanistic investigation for future studies.

An important aspect of our work is the identification of an endogenous target for MFH/Foxp1. Overexpression of Foxp1 has been shown to repress transcription of the SV40 promoter (25), the IL-2 promoter (25), and lung-specific CC10 promoter (20) based on promoter reporter assays. However, no data have been presented to date on the effect of Foxp1 on endogenous gene expression. Using retroviral overexpression of $M F H / F o x p 1$ in HL60 cells, we have identified the first physiologic target gene, $c-f m s$, for Foxp1 (Figure 5). We have pinpointed candidate MFH/Foxp1 binding sites in the c-fms promoter (-115 CAAGGCTTTGTTTTCTTCTTAA -94 and -13 GGAAAACAAGACAAACAGCC +7) that regulate $c$-fms expression. Repression of $c$-fms by MFH/Foxp1 appears to modulate important macrophage functions, as evidenced by our observation that overexpression of $M F H / F o x p 1$ markedly reduced adhesion and phagocytosis (Figure 4). Furthermore, identification of $c$-fms as an $\mathrm{MFH} /$ Foxp 1 target will provide important leads in establishing the mechanism(s) of Foxp-mediated transcriptional repression.

The monocyte-specific expression of $c$-fms is regulated by three transcription factors interacting with critical regions of the $c$-fms promoter, including PU.1, AML1 (CBF 2 2), and C/EBP (2). The $\mathrm{MFH} /$ Foxp1 binding sites identified in this study are immediately adjacent to the C/EBP and PU.1 binding sites, respectively (Figure $10)$. It is known that $\mathrm{C} / \mathrm{EBP} \alpha$ and the AML1 heterodimer synergisti-
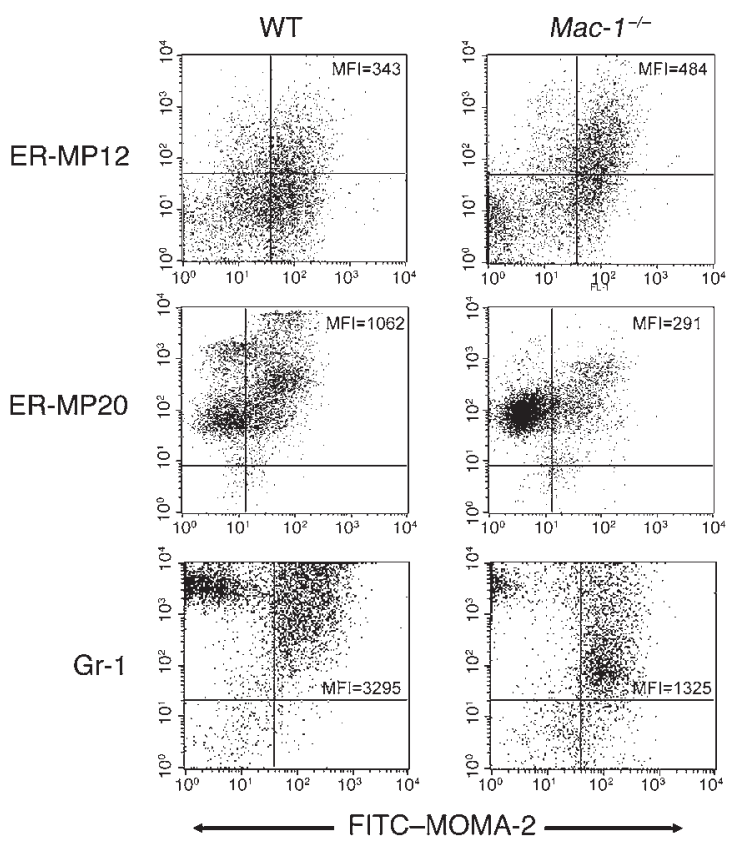
Table 1

Flow cytometric analysis of thioglycollate-induced peritoneal cells

\begin{tabular}{lccc} 
Double staining & Wild-type & Mac-1-deficient & $\boldsymbol{P}$ value \\
MOMA-2+/ER-MP12+ & $338 \pm 58$ & $411 \pm 63$ & 0.21 \\
MOMA-2+/ER-MP20+ & $907 \pm 169$ & $501 \pm 144$ & 0.029 \\
MOMA-2+/Gr-1+ & $3,740 \pm 628$ & $2,094 \pm 484$ & 0.002 \\
\hline
\end{tabular}

Peritoneal cells from wild-type and Mac-1-deficient mice were harvested and double-stained with FITC-conjugated MOMA-2, biotinylated ER-MP12 or ER-MP20, and PE-conjugated Gr-1 antibodies. ER-MP12 and ER-MP20 were stained using streptavidin-PE-cyanine5. Values represent mean fluorescence intensity found in gated wild-type and Mac-1-deficient MOMA-2+ population (mean $\pm \mathrm{SD}, n=3-5$ ).

cally activate the promoter (2), which raises the notion that physical interactions among $M F H / F o x p 1, \mathrm{C} / \mathrm{EBP} \alpha, \mathrm{AML} 1$, and/or PU.1 may be responsible for repression. Further studies are underway to dissect the nature of the inhibitory protein-protein interactions between MFH/Foxp1 and the known activators of $c$-fms expression.

$\mathrm{MFH} /$ Foxp 1 contains a glutamine-rich region at the aminoterminal end and a putative zinc-finger domain. Glutamine-rich regions such as these have been implicated as transcriptional activation/repression domains in other transcription factors $(37,38)$. Zinc-finger motifs have been implicated in transcriptional repression, most notably in the cases of YY1 and AEBP2 (39). Recent studies have reported that an independent transcription-repressor domain of murine Foxp1, which is highly homologous to human $M F H / F o x p 1$, lies in the $\mathrm{N}$-terminal end of the protein spanning the zinc-finger and leucine-zipper domains $(20,25)$. In contrast, in our study using human $M F H / F o x p 1$, deletion analysis suggests that transcription repression requires the glutamine-rich as well as the DNA-binding domains (Figure 6A). Several possibilities might explain the apparent discrepancy. First, there are significant structural differences between mouse and human Foxp1. Mouse Foxp1 contains an expanded polyglutamine tract (amino acid residues $78-108)$, which results in a larger mouse protein $(\sim 90 \mathrm{kDa})$ compared with the human protein $(\sim 85 \mathrm{kDa})$. Second, our deletion analyses have focused on a physiologic target gene (i.e., $c$-fms) rather than the nonphysiologic SV40 promoter (25) or putative physiologic targets (e.g., IL-2 and CC10 promoters). Third, cellspecific effects are likely operative, as evidenced by the prior report of Wang and coworkers that the activator/repressor activity of the glutamine region differs in Jurkat compared with 293T cells (25).

The intensity of inflammation is determined by both the extent of leukocyte recruitment and the proinflammatory actions of infiltrating leukocytes. Mac-1 regulates important leukocyte functions including migration, phagocytosis, and oxidative burst. Targeting leukocyte receptors like Mac-1 interrupts the adhesive and migratory capability of leukocytes and reduces tissue injury in models of inflammation (40). This study now provides in vivo evidence that Mac-1 regulates the expression of $M F H / F o x p 1$ and monocyte maturation (Figure 8). Mac-1-dependent signals promoting downregulation of $M F H / F o x p 1$ and, in turn, monocyte differentiation are therefore an obvious target for a new type of anti-inflammatory treatment.

\section{Methods}

Biological reagents and expression vectors. Anti-CD11b mAb included LPM19c (DAKO Corp., Carpinteria, California, USA), directed to the $\alpha_{M} I$ domain and capable of blocking fibrinogen binding (41). The stimulating anti-
CD18 mAb KIM127 (15) was a gift of M. Robinson (CellTech, Slough, United Kingdom). Anti- $\alpha$-tubulin and -Flag-tag mAb's were purchased from Sigma-Aldrich (St. Louis, Missouri, USA) and polyclonal anti-c-myc and anti-c-fms from Santa Cruz Biotechnology Inc. (Santa Cruz, California, USA). Flow cytometry mAb's included FITC-conjugated MOMA-2 (Serotec Inc., Raleigh, North Carolina, USA), r-phycoerythrin-conjugated (r-PE-conjugated) Gr-1 (BD Biosciences, San Jose, California, USA), and biotinylated ER-MP12 and ER-MP20 (Bachem Biosciences Inc., Torrance, California, USA). Anti-MFH/Foxp1 polyclonal antibody was raised against the carboxyl-terminal sequence CDHDRDYEDEPVNEDME and purified by affinity chromatography (Zymed Laboratories Inc., South San Francisco, California, USA). TGF- $\beta 1$ and $1,25-(\mathrm{OH})_{2}$ vitamin $\mathrm{D}_{3}$ were from EMB Biosciences Inc. (San Diego, California, USA). Elongase enzyme mix, SuperScript II reverse transcriptase, Lipofectamine, and terminal deoxynucleotidyl transferase (TdT) were from Invitrogen Corp. (Carlsbad, California, USA). Oligo primers were synthesized by Annovis Inc. (Acton, Pennsylvania, USA) and Invitrogen Corp. TnT Quick Coupled Transcription/Translation System and Luciferase Assay Substrate were from Promega Corp. (Madison, Wisconsin, USA). Human fibrinogen depleted of plasminogen, vWF, and fibronectin was obtained from Enzyme Research Laboratories (South Bend, Indiana, USA). PMA and RA were purchased from EMB Biosciences Inc. Expression vector pcDNA3.1/myc-his(-)A was from Invitrogen Corp. pGEM-T-Easy served as a cloning vector (Promega Corp.). Luciferase reporter constructs containing the $1.1-\mathrm{kb}$ proximal region of the human macrophage migration inhibitory factor promoter (R. Bucala, Yale University, New Haven, Connecticut, USA), the $1.35-\mathrm{kb}$ proximal region of the mouse PPAR $\gamma$-1 promoter (J.K. Reddy, Northwestern University, Chicago, Illinois, USA), the $0.49-\mathrm{kb}$ proximal region of the human $c$-fms promoter (D. Tenen, Beth Israel Deaconess Medical Center, Boston, Massachusetts, USA), and the $0.49-\mathrm{kb}$ proximal region of the human PU.1 promoter (D. Tenen) were obtained from the indicated sources. The expression construct

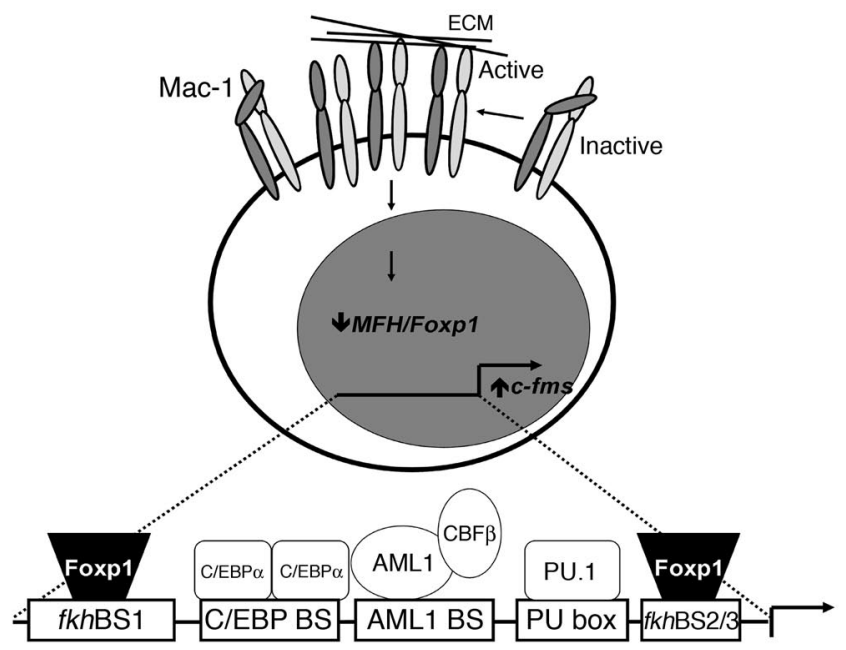

\section{Figure 10}

Schematic diagram of a proposed model for Mac-1-dependent regulation of the $c$-fms promoter and monocyte differentiation. Clustering of Mac-1 leads to downregulation of the transcriptional repressor MFH/ Foxp1. The monocyte-specific expression of $c$-fms is regulated by at least three transcription factors interacting with critical regions of the c-fms promoter: PU.1, AML1, and C/EBP (2). The $c$-fms promoter contains MFH/Foxp1 binding sites spanning -108 TTGTTT -103 and -13 GGAAAACAAGACAAACAGCC +7. These binding sites are immediately adjacent to the $\mathrm{C} / \mathrm{EBP} \alpha$ and PU.1 binding sites, respectively. Forkhead binding sites are indicated by $f k h \mathrm{BS} 1$ and $f k h \mathrm{BS} 2 / 3$. 
for PU.1 was obtained from T. Oikawa (Sasaki Institute, Tokyo, Japan), and the expression construct for $\mathrm{C} / \mathrm{EBP} \alpha$ was obtained from $\mathrm{D}$. Tenen.

Cell lines. HeLa, NIH 3T3, and 293 cells (American Type Culture Collection [ATCC], Manassas, Virginia, USA) were maintained in DMEM supplemented with $10 \%$ FBS. THP-1 monocytic cells (ATCC) were maintained in RPMI-1640 supplemented with 10\% FBS plus 2-mercaptoethanol (20 $\mu \mathrm{M})$. The human myeloblastic leukemia HL60 cell line (ATCC) was maintained in RPMI-1640 supplemented with 10\% FBS. HL60 cells were treated with PMA $(10 \mathrm{nM})$ to induce monocytic differentiation (42) or with RA (100 nM) to induce granulocytic differentiation (22).

Isolation of human monocytes. Human monocytes were isolated from peripheral blood by Ficoll-Hypaque centrifugation (43). Cell viability was greater than $95 \%$ as assessed by trypan blue exclusion, and monocyte purity was approximately $90 \%$ as determined by nonspecific esterase staining.

Adhesion assays. THP- 1 cells treated with cytokines ( $1 \mathrm{ng} / \mathrm{ml} \mathrm{TGF-} \beta 1$ and $50 \mathrm{nmol} / 1$ 1,25-(OH $)_{2}$ vitamin $\left.\mathrm{D}_{3}\right)$ were used for adhesion to immobilized fibrinogen exactly as previously reported (12). For inhibitor studies, adhesion was induced with the $\beta_{2}$-integrin-stimulating $\mathrm{KIM} 127 \mathrm{mAb}(5 \mu \mathrm{g} / \mathrm{ml})$, and 15 minutes later one of the following inhibitors (EMB Biosciences Inc.) was added: $250 \mathrm{nmol} / \mathrm{l}$ wortmannin, $100 \mathrm{nmol} / 1 \mathrm{SB} 203580,1 \mu \mathrm{mol} / \mathrm{l}$ herbimycin A, or $5 \mu \mathrm{mol} / 1$ bortezomib/PS-341 (Millennium Pharmaceuticals Inc., Boston, Massachusetts, USA). The addition of inhibitors 15 minutes after stimulation with KIM127 had no effect on the extent of adhesion.

Thioglycollate peritonitis model. Inflammatory cells from wild-type and Mac-1-deficient (27) C57BL/J6 mice were harvested and purified from the peritoneal cavity 16 hours after the intraperitoneal injection of $1 \mathrm{ml}$ sterile $3 \%$ thioglycollate broth (44). Animal care and procedures were reviewed and approved by the Harvard Medical School Standing Committee on Animals and performed in accordance with the guidelines of the American Association for Assessment and Accreditation of Laboratory Animal Care and the NIH.

Flow cytometry. Peritoneal cells were washed in FACS buffer and incubated with Fc Block (CD16/CD32; BD Biosciences) for 5 minutes before the addition of the appropriate $\mathrm{mAb}$ 's. Cell surface fluorescence was determined using directly FITC- and r-PE-conjugated mAb's. For biotinylated primary mAb's (ER-MP12 and ER-MP20), streptavidin-PE-cyanine5 (BD Biosciences) was used in double-staining experiments. Appropriate isotype controls were also included. FACS analyses were also performed to assess the expression of CD11b on the surface of transfected HL60 cells. Monoclonal antibodies included PE-conjugated LPM19c or isotype control. Events were recorded from samples using a FACScan (BD Biosciences Immunocytometry Systems, San Jose, California, USA) and analyzed using CellQuest (BD Biosciences - Immunocytometry Systems).

$D D$-PCR. DD-PCR was carried out using RNAmap kit, an mRNA DDPCR system (GenHunter Corp., Nashville, Tennessee, USA). Total RNA was extracted from both Mac-1-clustered and -nonclustered cells using RNeasy Mini Kit (QIAGEN Inc., Valencia, California, USA). RNA was then treated by DNase I, Amplification Grade (Invitrogen Corp.) and then purified by phenol/chloroform extraction. Total RNA was then reverse-transcribed by SuperScript II reverse transcriptase into cDNA. PCR reactions directed by different combinations of primers were performed and displayed in $6 \%$ polyacrylamide gels. For each primer combination, triplicate samples from three independent RNA pools were examined. cDNA probes of interest were cut from the gel, eluted, amplified again with the same primer, and then purified for further use in Northern analysis or RACE.

Northern analysis. THP-1 and HL60 cell mRNA was prepared from total RNA with Oligotex mRNA kit (QIAGEN Inc.), subjected to formaldehyde gel electrophoresis, and transferred to Duralon-UV membrane (Stratagene, La Jolla, California, USA). Filters were then hybridized with ${ }^{32} \mathrm{P}-$ labeled cDNA probes in QuikHyb solution (Stratagene).
RACE. mRNA purified from THP-1 cells was reverse-transcribed by SuperScript II reverse transcriptase, and the first-strand cDNA was then tailed by TdT in the presence of dATP. RACE reaction was then carried out using an oligo-dT primer, ATAGCGGCCGCTCGAGTTTTTTTTTTTTTT $\mathrm{T}(\mathrm{A} / \mathrm{C} / \mathrm{G})$, and an internal primer with sequence derived from the $12 \mathrm{CC} 4$ DD-PCR probe as well as primary RACE products. Among a series of designed primers, antisense primers with sequences TCAGCCCTCGTTAACTCCCTTAACAAG (nucleotides 3,860-3,833), TGGCAGGACGTCACGTCTGTGTGAGAG $(3,360-3,334)$, CGTGTACAGGATGCACGGCTTGCATAG (2,334-2,308), CGGAAGTAGGCAAACATTCGTGTGAACCAGTT $(1,939-1,908)$, and GAAGAGCTGGTTGTTTGTCATTCCTCTAGGGA (711-680) achieved the whole sequence upstream, whereas primer GGTCCCAAACATGGTATGCAGAAATGTG $(3,993-4,020)$ generated sequence downstream from the DD-PCR probe $(3,732-4,027)$. Primers GACCCCAGGCTTTTATGGCTGTGAGACACG (nucleotides 316-344) and TTGCCTCCTTTGATCAACAATAAGAGG $(4,276-4,250)$ were used to produce sequence bracketing the open reading frame of $12 \mathrm{CC} 4$.

Retroviral transduction. For retroviral studies, cDNA was cloned into the retroviral vector MSCV.GFP (gift of K. Murphy, Washington University, St. Louis, Missouri, USA), and retrovirus was generated as previously described (45). For infection of HL60 cells, retroviral supernatant and culture medium (DMEM containing $10 \%$ FCS plus $4 \mu \mathrm{g} / \mathrm{ml}$ polybrene) were mixed at a 1:1 ratio and added to $2 \times 10^{6} \mathrm{HL} 60$ EcoR cells (gift of G. Nolan, Stanford University, Stanford, California, USA). Within 72 hours, about $30-40 \%$ infectivity was noted by assessment for GFP. Cells underwent flow sorting for GFP-positivity, resulting in a more than 99\% GFP-positive population.

In vitro transcription and translation. In vitro transcription and translation of DNA was performed using TnT Quick Coupled Transcription/Translation System (Promega Corp.), according to manufacturer protocol.

Immunoblotting. Protein samples were denatured by boiling in SDS sample buffer, run on $8-12 \%$ reducing SDS-PAGE, and transferred to nitrocellulose. The membrane was then blotted with indicated antibody, and the bands were visualized with HRP-conjugated secondary antibody followed by the ECL Western blotting detection system (NEN Life Science Products Inc., Boston, Massachusetts, USA).

Phagocytosis assay. Twelve hours after PMA treatment, GFP-expressing HL60 cells $\left(1 \times 10^{6}\right.$ cells $\left./ \mathrm{ml}\right)$ were cultured in the presence or absence of Texas red-conjugated zymosan A BioParticles (Molecular Probes Inc., Eugene, Oregon, USA) for 2 hours at $37^{\circ} \mathrm{C}$. Cells were viewed for internalization of the particles by fluorescence microscopy after quenching of extracellular fluorescence with trypan blue. Assays were performed in triplicate from three independent experiments.

MFH/Foxp1 deletion mutants. To map the repression domain, a series of $\mathrm{N}$ - and C-terminal MFH/Foxp1 deletion mutants were generated. MFH/ Foxp1 deletion mutants were obtained by PCR using combinations of the primers listed below and then cloned, in-frame, into NotI-BamHI sites of pcDNA3.1/myc-his(-)A (Invitrogen Corp.): (a) full-length MFH/Foxp1 (amino acids 1-677): CTTGCGGCCGCTACCATGGGACAAGAATCTGGGACTGAGACAAAG; (b) MFH/Foxp1 without Q-rich domain (amino acids 224-677): CTTGCGGCCGCTACCATGGGAATTCCAACAGAACTGCAGCAGCTC; (c) MFH without Q-rich and zinc-finger domains (amino acids 367-677): CTTGCGGCCGCTACCATGGGACACACGGTGGGACCCATCCGCAGG; (d) Q-rich domain of MFH (amino acids 1-222): AGTGGGATCCCTTGCCTTGAGCAAGAGGTTGAAGGGG; (e) Q-rich and zinc-finger domains of MFH (amino acids 1-428): AGTGGGATCCCTTGCTGGTGGTTGTGATGACAGAGGA; and (f) 3'-terminal primer: AGTGGGATCCCTTCTCCATGTCCTCGTTTACTGGTTC.

Site-directed mutagenesis. The candidate Foxp1 binding sites of the $c$-fms luciferase promoter construct spanning -175 to +71 were mutated using the QuikChange mutagenesis kit (Stratagene). These mutations changed 
the wild-type sequence $5^{\prime}-108$ TTGTTTTC $-1013^{\prime}$ to ggaTTTTC, TTGagaTC, or TTGTTccg and also changed the wild-type sequence $5^{\prime}-13$ GGAAAACAAGACAAACAGCC +7 to GGAtgcagAGACgtcgtGCC.

Reporter assays. NIH 3T3 cells were transfected on a 12-well plate using Lipofectamine (Invitrogen Corp.) with $0.5 \mu \mathrm{g}$ luciferase promoter reporter constructs, $5.5 \mu \mathrm{g}$ pcDNA3.1/myc-his(-)A empty vector, pcDNA3.1/mychis(-)A-MFH/Foxp1 or indicated mutants, and $0.05 \mu \mathrm{g}$ pCMV- $\beta-G a l$ (Promega Corp.) per well (triplicate samples per treatment group). HeLa cells were transfected by calcium phosphate precipitation method using $0.25 \mu \mathrm{g}$ pCMV- $\beta$-Gal, $1.0 \mu \mathrm{g}$ luciferase promoter reporter constructs, and $4.5 \mu \mathrm{g}$ pcDNA3.1/myc-his(-)A-MFH/Foxp1. After 48-72 hours, transfected cells were lysed for measurement of luciferase activity. $\beta$-Galactosidase activity was analyzed with High-Sensitivity $\beta$-Gal Assay Kit (Stratagene). Luciferase activities were determined and normalized on the basis of $\beta$-galactosidase activity. All transfections were performed in triplicate from at least three independent experiments.

Semiquantitative RT-PCR. Semiquantitative RT-PCR was performed as previously described (46). The following primers were used: MFH/Foxp1 (sense, 5'-GTCGGGCGGCAGCAACCACTTACTAGAGTG-3'; antisense, 5'-GAAGAGCTGGTTGTTTGTCATTCCTCTTGGGA-3'); MFH/Foxp1myc (sense, 5'-TGCAGCTTTACAGGCTTCAATGGC-3'; antisense, 5'TAGAAGGCACAGTCGAGG-3'); c-fms (sense, 5'-GCATCCTCAGCACCAACAAC-3'; antisense, 5'-TCCCAGGTAGGTCCAGTTAA-3'); GAPDH (sense, 5'-GGCGCTGAGTACGTCGTGGAGTCCACTG-3'; antisense, 5'CAGCCCCAGCGTCAAAGGTGGAGGAGTG-3').

Production of GST fusion protein of MFH/Foxp1. Generation of a GST fusion protein for the DNA-binding domain of $M F H / F o x p 1$ was done by inframe cloning of DNA sequences corresponding to amino acids 431-559 of the gene into pGEX-4T-1 vector (Amersham Biosciences, Piscataway, New Jersey, USA) with PCR primers CCGCGTGGATCCACGGTGGGACCCATCCGCAGGCGG and CACGATGCGGCCGCTCGTTTTTA-
ATAAGGGAAGGGTTACC. Constructs were sequenced, and the in-frame positive ones were used to transform competent BL21-CodonPlus-RP Escherichia coli (Stratagene). Miniprep proteins were displayed by SDS-PAGE and visualized by staining with Coomassie blue. High-expressing colonies were selected for large scale preparation of the fusion protein.

EMSA. The GST-MFH/Foxp1 DNA-binding domain (amino acids 431$559, \sim 10 \mu \mathrm{g}$ ) was incubated with $0.5 \mathrm{ng}{ }^{32} \mathrm{P}$-end-labeled $c$-fms promoter oligonucleotides and $1.0 \mu \mathrm{g}$ of poly(dI-dC). Oligonucleotide-protein complexes were then resolved by $4 \%$ native PAGE gel in high-ionic-strength buffer ( $45 \mathrm{mmol} / 1$ Tris, pH 8.3, $45 \mathrm{mmol} / \mathrm{l}$ boric acid, $1 \mathrm{mmol} / \mathrm{l}$ EDTA). Dried gels were subjected to autoradiography. Oligonucleotides corresponding to forkhead-like binding sites of the $c$-fms promoter, and their corresponding antisense strands, were synthesized: wild-type -115 CAAGGCTTTGTTTTCTTCTTAA -94 and three mutants (mutated bases in lower case), -115 CAAGGCTggaTTTTCTTCTTAA -94, -115 CAAGGCTTTGagaTCTTCTTAA -94, and -115 CAAGGCTTTGTTccgTTCTTAA -94; and wild-type -15 GAGGAAAACAAGACAAACAGCCAGT +10 and mutant - 15 GAGGAtgcagAGACgtcgtGCCAGT + 10 .

\section{Acknowledgments}

This research was supported by NIH grants R01 HL57506, R01 DK55656, and R01 HL73852 (to D.I. Simon), R01 HL03747 and R01 HL075427 (to M.K. Jain), and K08 HL67755 (to M.W. Feinberg).

Received for publication January 16, 2004, and accepted in revised form June 15, 2004.

Address correspondence to: Daniel I. Simon, Cardiovascular Division, Brigham and Women's Hospital, 75 Francis Street Tower 3, Boston, Massachusetts 02115, USA . Phone: (617) 525-4371; Fax: (617) 525-4377; E-mail: dsimon@rics.bwh.harvard.edu.
1. Tenen, D.G., Hromas, R., Licht, J.D., and Zhang, D.E. 1997. Transcription factors, normal myeloid development, and leukemia. Blood. 90:489-519.

2. Zhang, D.E., et al. 1996. CCAAT enhancer-binding protein $(\mathrm{C} / \mathrm{EBP})$ and AML1 (CBF alpha2) synergistically activate the macrophage colonystimulating factor receptor promoter. Mol. Cell. Biol. 16:1231-1240.

3. Wiktor-Jedrzejczak, W., et al. 1990. Total absence of colony-stimulating factor 1 in the macrophagedeficient osteopetrotic (op/op) mouse. Proc. Natl. Acad. Sci. U. S. A. 87:4828-4832.

4. Dai, X.M., et al. 2002. Targeted disruption of the mouse colony-stimulating factor 1 receptor gene results in osteopetrosis, mononuclear phagocyte deficiency, increased primitive progenitor cell frequencies, and reproductive defects. Blood. 99:111-120.

5. Valledor, A.F., Borras, F.E., Cullell-Young, M., and Celada, A. 1998. Transcription factors that regulate monocyte/macrophage differentiation. J. Leukoc. Biol. 63:405-417.

6. Hynes, R.O. 1992. Integrins: versatility, modulation, and signaling in cell adhesion. Cell. 69:11-25.

7. Giancotti, F.G. 1997. Integrin signaling: specificity and control of cell survival and cell cycle progression. Curr. Opin. Cell Biol. 9:691-700.

8. Fan, S.-T., and Edgington, T.S. 1991. Coupling of the adhesive receptor CD11b/CD18 to functional enhancement of effector macrophage tissue factor response. J. Clin. Invest. 87:50-57.

9. Rezzonico, R., Chicheportiche, R., Imbert, V., and Dayer, J.M. 2000. Engagement of CD11b and CD11c beta2 integrin by antibodies or soluble CD23 induces IL-1beta production on primary human monocytes through mitogen-activated protein kinase-dependent pathways. Blood. 95:3868-3877.
10. Sitrin, R.G., Pan, P.M., Srikanth, S., and Todd, R.F., 3rd. 1998. Fibrinogen activates NF-kappa B transcription factors in mononuclear phagocytes. J. Immunol. 161:1462-1470.

11. Bianchi, E., et al. 2000. Integrin LFA-1 interacts with the transcriptional co-activator JAB1 to modulate AP-1 activity. Nature. 404:617-621.

12. Shi, C., Zhang, X., Chen, Z., Robinson, M.K., and Simon, D.I. 2001. Leukocyte integrin Mac-1 recruits toll/interleukin-1 receptor superfamily signaling intermediates to modulate NF-kappaB activity. Circ. Res. 89:859-865.

13. Rogers, C., Edelman, E.R., and Simon, D.I. 1998. A monoclonal antibody to the $\beta 2$-leukocyte integrin Mac-1 (CD11b/CD18) reduces intimal thickening after angioplasty or stent implantation in rabbits. Proc. Natl. Acad. Sci. U. S. A. 95:10134-10139.

14. Simon, D.I., et al. 2000. Decreased neointimal formation in Mac-1(-/-) mice reveals a role for inflammation in vascular repair after angioplasty. J. Clin. Invest. 105:293-300.

15. Robinson, M.K., et al. 1992. Antibody against the Leu-CAM beta-chain (CD18) promotes both LFA-1and CR3-dependent adhesion events. J. Immunol. 148:1080-1085.

16. Diamond, M.S., Garcia-Aguilar, J., Bickford, J.K., Corbi, A.L., and Springer, T.A. 1993. The I domain is a major recognition site on the leukocyte integrin Mac-1 (CD11b/CD18) for four distinct adhesion ligands. J. Cell Biol. 120:1031-1043.

17. Li, C., and Tucker, P.W. 1993. DNA-binding properties and secondary structural model of the hepatocyte nuclear factor 3 /fork head domain. Proc. Natl. Acad. Sci. U. S. A. 90:11583-11587.

18. Lai, E., Clark, K.L., Burley, S.K., and Darnell, J.E., Jr. 1993. Hepatocyte nuclear factor 3/fork head or "winged" proteins: a family of transcription factors of diverse biologic function. Proc. Natl. Acad. Sci. U. S. A. 90:10421-10423.

19. Banham, A.H., et al. 2001. The FOXP1 winged helix transcription factor is a novel candidate tumor suppressor gene on chromosome 3 p. Cancer Res. 61:8820-8829.

20. Shu, W., Yang, H., Zhang, L., Lu, M.M., and Morrisey, E.E. 2001. Characterization of a new subfamily of winged-helix/forkhead (Fox) genes that are expressed in the lung and act as transcriptional repressors. J. Biol. Chem. 276:27488-27497.

21. Rovera, G., Santoli, D., and Damsky, C. 1979. Human promyelocytic leukemia cells in culture differentiate into macrophage-like cells when treated with a phorbol diester. Proc. Natl. Acad. Sci. U. S. A. 76:2779-2783.

22. Grande, A., et al. 1995. All-trans-retinoic acid induces simultaneously granulocytic differentiation and expression of inflammatory cytokines in HL-60 cells. Exp. Hematol. 23:117-125.

23. Yan, S.R., and Berton, G. 1998. Antibodyinduced engagement of beta 2 integrins in human neutrophils causes a rapid redistribution of cytoskeletal proteins, Src-family tyrosine kinases, and $\mathrm{p} 72$ syk that precedes de novo actin polymerization. J. Leukoc. Biol. 64:401-408.

24. Hideshima, T., Chauhan, D., Schlossman, R., Richardson, P., and Anderson, K.C. 2001. The role of tumor necrosis factor alpha in the pathophysiology of human multiple myeloma: therapeutic applications. Oncogene. 20:4519-4527.

25. Wang, B., Lin, D., Li, C., and Tucker, P. 2003. Multiple domains define the expression and regulatory properties of foxp 1 forkhead transcriptional repressors. J. Biol. Chem. 278:24259-24268.

26. Coxon, A., et al. 1996. A novel role for the beta 2 integrin CD11b/CD18 in neutrophil apoptosis: a 
homeostatic mechanism in inflammation. Immunity. 5:653-666.

27. Lu, H., et al. 1997. LFA-1 is sufficient in mediating neutrophil emigration in Mac-1-deficient mice. J. Clin. Invest. 99:1340-1350.

28. Henkel, G.W., McKercher, S.R., Leenen, P.J., and Maki, R.A. 1999. Commitment to the monocytic lineage occurs in the absence of the transcription factor PU.1. Blood. 93:2849-2858.

29. Ling, V., et al. 1997. Structural identification of the hematopoietic progenitor antigen ER-MP12 as the vascular endothelial adhesion molecule PECAM-1 (CD31). Eur. J. Immunol. 27:509-514.

30. McCormack, J.M., Leenen, P.J., and Walker, W.S. 1993. Macrophage progenitors from mouse bone marrow and spleen differ in their expression of the Ly-6C differentiation antigen. J. Immunol. 151:6389-6398.

31. de Bruijn, M.F., et al. 1994. Distinct mouse bone marrow macrophage precursors identified by dif ferential expression of ER-MP12 and ER-MP20 antigens. Eur. J. Immunol. 24:2279-2284.

32. Kraal, G., Rep, M., and Janse, M. 1987. Macrophages in $\mathrm{T}$ and $\mathrm{B}$ cell compartments and other tissue macrophages recognized by monoclonal antibody MOMA-2. An immunohistochemical study. Scand. J. Immunol. 26:653-661.
33. Hestdal, K., et al. 1991. Characterization and regulation of RB6-8C5 antigen expression on murine bone marrow cells. J. Immunol. 147:22-28.

34. Zhang, W., and Kone, B.C. 2002. NF-kappaB inhibits transcription of the $\mathrm{H}(+)-\mathrm{K}(+)$-ATPase alpha(2)subunit gene: role of histone deacetylases. Am. J. Physiol. Renal Physiol. 283:F904-F911.

35. Novitskiy, G., Potter, J.J., Rennie-Tankersley, L., and Mezey, E. 2004. Identification of a novel NF-kappaB-binding site with regulation of the murine alpha2(I) collagen promoter. J. Biol. Chem. 279:15639-15644.

36. Campbell, K.J., Rocha, S., and Perkins, N.D. 2004 Active repression of antiapoptotic gene expression by RelA(p65) NF-kappa B. Mol. Cell. 13:853-865.

37. Madden, S.L., Cook, D.M., and Rauscher, F.J., 3rd. 1993. A structure-function analysis of transcriptional repression mediated by the WT1, Wilms' tumor suppressor protein. Oncogene. 8:1713-1720.

38. Gerber, H.P., et al. 1994. Transcriptional activation modulated by homopolymeric glutamine and proline stretches. Science. 263:808-811.

39. He, G.P., Kim, S., and Ro, H.S. 1999. Cloning and characterization of a novel zinc finger transcriptional repressor. A direct role of the zinc finger motif in repression. J. Biol. Chem. 274:14678-14684.

40. Simpson, P.J., et al. 1988. Reduction of experimen- tal myocardial reperfusion injury by a monoclonal antibody (anti Mo1, anti CD11b) that inhibits leukocyte adhesion. J. Clin. Invest. 81:624-629.

41. Diamond, M., Garcia-Aguiliar, J., Bickford, J. Corbi, A., and Springer, T. 1993. The I domain is a major recognition site on the leukocyte integrin Mac-1 (CD11b/CD18) for four distinct ligands. J. Cell Biol. 120:1031-1043.

42. Nguyen, H.Q., Hoffman-Liebermann, B., and Liebermann, D.A. 1993. The zinc finger transcription factor Egr-1 is essential for and restricts differentiation along the macrophage lineage. Cell. 72:197-209.

43. Fogelman, A.M., Haberland, M.E., Seager, J., Hokom, M., and Edwards, P.A. 1981. Factors regulating the activities of the low density lipoprotein receptor and the scavenger receptor on human monocyte-macrophages. J. Lipid Res. 22:1131-1141.

44. Simon, D.I., et al. 2000. Platelet glycoprotein ibalpha is a counterreceptor for the leukocyte integrin Mac-1 (CD11b/CD18). J. Exp. Med. 192:193-204.

45. Ranganath, S., et al. 1998. GATA-3-dependent enhancer activity in IL-4 gene regulation. J. Immunol. 161:3822-3826.

46. Kuwata, T., et al. 2000. Vitamin A deficiency in mice causes a systemic expansion of myeloid cells. Blood. 95:3349-3356. 